\title{
Leader
}

\section{The risks of infection transmission by blood transfusion in England}

\author{
Kate Soldan, John Barbara
}

Strategies implemented to prevent transmission of infections by blood transfusion have been very successful. Despite this, the risk of infectious donations entering the blood supply and transmitting infection to the recipients of blood components and blood products is continually under scrutiny.

In order to assess the risks and consequences of transfusion transmitted infections the characteristics of blood borne infections, of donations, and of blood recipients need to be considered. Over the years, knowledge about new agents and about potential failures in the strategies to exclude known agents has increased. Consequently the range of possible strategies to exclude infections from the blood supply has also increased, and debate about the risks of infection transmission by blood transfusion has become more complex.

\section{Transfusion transmissible infections}

At certain stages in their natural history many viral, bacterial, and protozoal infections can be blood borne and may be transmitted by transfusion. Fortunately for transfusion medicine, many blood borne organisms cause symptoms that render their victims too unwell, or obviously unfit, to donate blood. Other agents are only present in the blood transiently and some agents do not survive the conditions of blood storage outside the human body.

Variations in the length of time over which agents are present in the blood, and are viable in stored blood, determine to a large extent the variations in the risk that infectious donations will be collected. Infections of most concern are those that have long periods of infectivity in the absence of any clinical signs or symptoms of infection and are stable in stored blood (for example, hepatitis B virus (HBV), HIV, and hepatitis $\mathrm{C}$ virus (HCV)). The length of time between infection and the development of detectable serological markers (the window period) also varies between agents (for example, 22 days for anti-HIV ${ }^{1}$ and 66 days for anti-HCV ${ }^{2}$ using current assays). The shorter the window period, relative to the total asymptomatic seropositive infective period, the better is the detection of infectious donations by serological testing (all other things being equal).

For infections with transient blood borne infectivity (for example, hepatitis A virus
(HAV) and parvovirus B19), the risk of infectious donations being collected depends upon the incidence of the infection in the donor population and the length of the infectious period.

\section{Strategies to reduce risk}

There are three main strategies for preventing infectious donations from entering the blood supply issued to hospitals. The first concerns the recruitment and selection of blood donors who do not have a known increased risk of infection. The second is the testing of donations for serological markers of infections. The third covers the control of cleanliness during component production.

Donor recruitment and selection aims to select a group of individuals with a low risk of infection: the prevalence of infection and the incidence of infection should both be low. In practice incidence is often difficult to measure. The selection of a "low risk" group therefore often depends on identifying groups with low seroprevalence and without the characteristics or exposures associated with an increased risk of infection. There are some general guidelines for donor selection (which are well founded in experience). Voluntary donors are considered safer than paid donors, and repeat donors safer than new donors. However, selection of these individuals is not guaranteed to be effectiveparticularly for new infections or for infections with changing epidemiology.

New knowledge about exposures of increased risk for blood borne infections is regularly considered so that guidelines for predonation donor selection in the United Kingdom can be revised as necessary. Inapparent infections and non-recognition or denial of risk factors in donors prevents the exclusion of all infected donations by predonation selection criteria.

Over the years there has been a steady introduction of available measures to reduce risks that have been recognised. Table 1 shows the tests for markers of transfusion transmissible infection that are currently performed on all blood donations in the United Kingdom. The introduction of each of these tests has led to a reduction in the number of transfusion transmitted infections (table 1). During the first full year of anti-HIV (1986) and anti-HCV (1992) testing in England and Wales, 38 and 807 
Table 1 Routine testing for markers of transfusion transmissible infection in England and Wales and the effect of testing on the prevention of infections in blood transfusion recipients

\begin{tabular}{|c|c|c|c|}
\hline Assay & $\begin{array}{l}\text { Date of introduction to } \\
\text { routine donation testing }\end{array}$ & $\begin{array}{l}\text { Number of positive } \\
\text { donations excluded by } \\
\text { testing during } 1997\end{array}$ & $\begin{array}{l}\text { Reduction in transfusion transmitted infections in } \\
\text { England and Wales following introduction of routine } \\
\text { test }{ }^{\star}\end{array}$ \\
\hline $\begin{array}{l}\text { Treponemal } \\
\text { antibodies }\end{array}$ & By 1950 & $\begin{array}{l}100 \\
\text { (1 in } 26703 \text { donations) }\end{array}$ & $\begin{array}{l}\text { Uncertain: it is difficult to ascribe reduction in } \\
\text { transfusion transmitted syphilis to testing since } \\
\text { storage at } 4^{\circ} \mathrm{C} \text { leads to inactivation of } T \text { pallidum }\end{array}$ \\
\hline HBsAg & Early 1970 s & $\begin{array}{l}123 \\
\text { ( } 1 \text { in } 21710 \text { donations })\end{array}$ & $\begin{array}{l}\text { There was a marked fall in post-transfusion acute } \\
\text { HBV infections: eg, North London blood centre } \\
\text { recorded } 30 \text { reports of cases in } 1970,12 \text { in } \\
1972,6 \text { in } 1974 \text {, and } 3 \text { in } 1976^{3}\end{array}$ \\
\hline Anti-HIV 1 & October 1985 & 29 & 69 HIV infections were diagnosed that were \\
\hline Anti-HIV 1 and 2 & June 1990 & ( 1 in 92079 donations) & $\begin{array}{l}\text { probably transmitted by transfusion in the UK } \\
\text { before } 10 / 85+\text {, and } 3 \text { that were transfused } \\
\text { between } 10 / 85 \text { and the end of } 1997 \text {. }\end{array}$ \\
\hline Anti-HCV & September 1991 & $\begin{array}{l}236 \\
\text { ( } 1 \text { in } 11315 \text { donations) }\end{array}$ & $\begin{array}{l}\text { Transfusion before } 9 / 91 \text { has been associated with } \\
128 \text { laboratory reports }(4.3 \%) \text { of } \mathrm{HCV} \text { infection } \\
\text { with risk factor information }(1992-1996) .^{4} \\
\text { Between } 1 / 10 / 95 \text { and } 30 / 6 / 98 \text {, one case of } \mathrm{HCV} \\
\text { transmission by transfusion post } 9 / 91 \text { has been } \\
\text { reported }\end{array}$ \\
\hline
\end{tabular}

*Other factors, such as improved donor selection, will have contributed.

+Source: PHLS AIDS Centre (data as of 1 September 1998).

¥Source: NBA/PHLS CDSC, unpublished infection surveillance report No 7.

HbsAg, hepatitis B surface antigen; HCV, hepatitis C virus; HIV, human immunodeficiency virus.

positive donors were identified, respectivelythus preventing the donations from these donors entering the blood supply. As time passes following the introduction of a marker test, and the population of repeat blood donors passes through the testing process, the overall rate of infectious donations identified decreases. The number of positive donations excluded from the blood supply in England and Wales by donation testing during 1997 is shown in table 1. Many, if not all, donations positive for HBsAg, anti-HIV, or anti-HCV are expected to lead to infection in at least one blood recipient if they are not excluded from the blood supply.

Maximising the effectiveness of donation testing includes assuring good test performance. This can be obtained by the evaluation of test kits, and test kit batches, for suitability and reliability in the blood centre setting, before their use by transfusion services. Monitoring performance once a test is in use is also important.

Testing blood donations improves the safety

of the blood supply in two direct ways:

- Infectious donations found to be positive for markers of infection at the time of donation are removed.

- Infected donors are excluded from the donor population, and infected donations are therefore prevented from entering blood centres in the future.

These factors can be quantified to assess the benefits of donation testing.

Testing also improves the safety of the blood supply in two indirect ways:

- Donors who are at increased risk of blood borne infections are excluded from the donor population. As blood borne infections often have common routes of transmission, donors with evidence of one infection may be at increased risk of having other blood borne infections that are not detected by donation testing. Also, some individuals who have been in contact with infected donors (for example, sexual contacts) may be at increased risk of infection, and infected donations may be prevented from entering blood centres if these individuals are instructed not to donate blood.

- The diagnosis of infection in a donor, and the surveillance of infections and risk factors in donors, can improve methods of donor selection-for example, the detection of $\mathrm{HCV}$ antibodies in blood donors revealed a large group of donors who had been exposed to blood borne infections by injecting drugs. ${ }^{5}$

The probability of transmitting an infection by blood transfusion can be reduced by certain manufacturing processes and conditions. Strict control of cleanliness during component production limits the opportunities for bacterial contamination. Storage of whole blood and red cells at $4 \pm 2{ }^{\circ} \mathrm{C}$ limits the growth of any bacteria that are present.

Developments to testing systems, and controls on those systems, that ensure the release only of negative components have been crucial in the improved safety gained by donation testing. Automation of testing, along with inclusion of controlled steps in commercial tests, has enabled strict standardisation and close monitoring of the testing process. One example of an important addition to the testing processes is sample addition monitors that change colour (measurable on a spectrophotometer) when serum or plasma is added. Another is process control automation. Use of appropriate quality control samples, as well as the manufacturer's controls and "go-no-go" samples, adds a further check on test performance. The computerisation of test results and of component release has helped to increase safety in the face of increasing numbers of donations and the increasing volume of data generated during the testing of each donation.

Practices beyond the transfusion centre also contribute to the prevention of transfusion transmitted disease. Strategies to avoid transfusion as a treatment unless absolutely necessary, and to inactivate viruses by heat or solvent detergent treatments of products, prevent exposures. Strategies to provide prophylactic 
treatment to recipients can also play a useful role. For example, HBV immunisation is currently recommended for haemophiliacs, those receiving regular blood transfusions or blood products, or those carers responsible for the administration of such products. ${ }^{6}$

Manufacturing processes that involve pooling donations or components, for example for treatment with solvent detergents, require careful consideration. Pooling (unless the infection is neutralised by antibodies also present in the pool) can lead to a single infectious donation entering multiple products, and is therefore avoided. Pooling is particularly dangerous with regard to agents that are not excluded by testing, including agents that are as yet unknown.

Additional serological tests are performed in some countries. Some detect infections missed by current testing-for example, HIV p24 antigen and anti-HBc. Others detect transfusion transmissible infections that are currently not tested for in the United Kingdom-for example, anti-HTLV. Others detect surrogate markers of infection-for example, alanine transaminase for hepatitis viruses, low $\mathrm{pH}$ haemagglutination for parvovirus B19, and alphaneopterin for detecting inflammation. The frequency of infections, and therefore the risk of transmissible infection, in donations in England and Wales has tended to be lower than in countries where additional tests have been adopted. However, this in not always the case. Factors such as the expected risk of disease occurring in recipients, the amount of public concern about blood safety and the infection in question, and the availability of resources have also played a part in determining the differences in blood testing strategies in different countries. The availability of tests for nucleic acids provides an opportunity to detect infections that cannot be detected by serological tests. Donations collected during the window period of early infection are the main candidates. Nucleic acid testing (NAT) should detect infectious donations from seronegative donors and from any seropositive donors that routine serological testing fails to detect. Nucleic acid tests are soon to be used for HCV RNA in mini-pools of plasma samples destined for pooled product manufacture. ${ }^{7}$ The potential additional benefit for a blood service of such procedures for specific agents will depend on the epidemiology of the agent in their population.

Assessing the value of additional donation testing strategies must consider some or all of the following costs:

- the cost of test kits and reagents and related laboratory costs including staff time;

- the costs of confirmatory testing on reactive donations;

- the costs of notifying, counselling, and referring donors who are positive to new tests, or who have persistent false reactivity to the new tests used;

- the costs of replacing donors excluded because of positivity (or false persistent reactivity) to the tests used;
- the costs of any delay in the release of blood components while testing is performed;

- the costs of added data management and added complexity to the blood release procedure;

- the costs of look-backs - that is, of tracing and testing recipients who may have been exposed to infection by other donations from donors found to be positive.

\section{Quantification of risks}

Quantifying the risk of transfusion transmission of infection can be attempted by several methods, each with different limitations (table 2).

Surveillance systems monitor diagnosed transfusion transmitted infections. Several factors common to transfusion transmitted infections, and to transfusion recipients, are likely to contribute to a lack of clinically apparent symptoms and therefore to underdiagnosis of infections. Other treatments may negate or modify symptoms - many transfusion recipients are receiving antibiotic drugs and are therefore less likely to suffer observable consequences from bacterial infections. Transfusion recipients are sick or injured, and often elderly, and have high mortality from other causes. The recipients who receive relatively large numbers of transfusions, and are therefore at the highest risk of transfusion transmitted infections, have the highest mortality rates. Long presymptomatic periods are common for persistent blood borne viruses. Whether infection with a larger viral dose, at an older age, and in already ill or immunocompromised individuals alters this period is not always known. Transfusion in the past may be overlooked as a possible route of infection when diagnosis is delayed for such long periods. Naturally acquired immunity to infection may be quite high for some infections, and asymptomatic infection is common in the young age groups with low levels of naturally acquired immunity (for example, HAV and B19). Recognised cases of transfusion transmitted infections are likely to be those with the more apparent, and more severe, clinical consequences.

These ascertainment biases and limitations can be overcome by actively following up transfused recipients and testing them for evidence of transfusion transmitted infections. In the United Kingdom, transfusion transmission of infection with observed clinical consequences is rare-both in absolute terms and relative to incidents of infection transmission by other routes. The number of recipients that need to be followed up in order to obtain a precise estimate of transmission rates is therefore very large, and such studies have become prohibitively expensive. A recent study of 21800 units found no transfusion transmitted HIV, HBV, HCV, or HTLV (human T cell leukaemia virus) I and II infections. ${ }^{8}$

Another approach is to estimate the number of infectious donations that current donation testing is not expected to detect. To attempt such estimation, information is needed about infection rates in the population donating blood, about the development and persistence 
Table 2 Sources of quantitative data and estimates in the United Kingdom about how many transfusion transmitted infections occur (or are reported) in one year (equivalent to per 2.5 million donations)

\begin{tabular}{llll}
\hline Infection & $\begin{array}{l}\text { Surveillance of apparent cases. } \\
\text { [Source: SHOT reports } \\
\text { received Oct'96-Sep'97*] }\end{array}$ & $\begin{array}{l}\text { Prospective study of transfusion } \\
\text { recipients. [Source: study of 21800 } \\
\text { donations 1996 } \\
\text { intervals) }\end{array}$ & $\begin{array}{l}\text { Estimated infectious donations released into } \\
\text { the blood supply. [Source: unpublished study } \\
\text { using 1993-1995 donor infection data for } \\
\text { England] (95\% confidence intervals) }\end{array}$ \\
\hline HAV & $1^{9}$ & NA & NA \\
HIV & $1\left(3\right.$ recipients infected) ${ }^{10}$ & $0(0$ to 423$)$ & $0.8(0.3$ to 2.5) \\
HCV & 1 & $0(0$ to 423$)$ & $11(9.4$ to 18.3) \\
HBV & 1 & $0(0$ to 423$)$ & NA \\
HTLV I and II & 0 & $0(0$ to 423$)$ & NA \\
Bacteria & 3 & NA & NA \\
\hline
\end{tabular}

NA, not available

* Source: Serious hazards of transfusion: Annual Report 1996-1997.

$\mathrm{HA}(\mathrm{B}, \mathrm{C}) \mathrm{V}$, hepatitis $\mathrm{A}(\mathrm{B}, \mathrm{C})$ virus; HIV, human immunodeficiency virus; HTLV, human $\mathrm{T}$ cell leukaemia virus.

of the markers that are tested for, and about the tests and the testing system used. The probability of a donation being collected during the window period when the tests used cannot detect evidence of infection depends upon the incidence of the infection and the length of the window period. The probability of symptoms that may prevent donation occurring during this period may also need to be considered. Incidence is usually calculated using observations of acute infections in donors or observations of seroconversions in repeat donors. The probability of a false negative test result depends upon the prevalence of the marker and the sensitivity of the test. The probability of a marker positive donation being released into the blood supply owing to a failure, or error, in the testing system also depends upon the prevalence of the marker and upon the probability of a failure or error.

Infectious donations do not necessarily mean morbidity in recipients, and estimating the effect of infectious donations requires knowledge about transmission and the natural history of infections.

Transfusion transmitted infections also bear a risk of onward transmission. The major risk factors for transmission of the persistent viral infections - that is, injecting drug use and sexual contact - may be relatively rare among transfusion recipients. However, this is not always the case and other types of contact - especially those common in health care settings-pose a risk of secondary transmission.

\section{Our limited knowledge}

When considering the infectivity of blood from donors, and the natural history of infections transmitted by transfusion, knowledge obtained from observing infections transmitted by other routes may not be reliable. In particular, the progression of disease caused by some viral infections may be affected by the viral load at the time of infection. An infected blood component typically exposes a recipient to a far higher viral dose than other routes of transmission.

Whether prion disease can be transmitted by transfusion is currently uncertain. ${ }^{11}$ Unknown infections and infections with increasing potential to cause harm to recipients owing to the changing epidemiology of the infection, or changing vulnerability of blood recipients to disease, may pose the greatest risks of infection to recipients. Avoidance of unnecessary transfusion and vigilance of blood borne infectious diseases in the general population and in blood recipients are therefore important general components of transfusion medicine.

We thank Mary Ramsay for comments on the manuscript.

1 Busch MP, Lee LL, Satten GA, et al. Time course of detection of viral and serological markers preceding human immunodeficiency virus type 1 seroconversion: implications for screening of blood and tissues donors. Transfusion 1995;35:91-7.

2 Barrera JM, Francis B, Ercilla G, et al. Improved detection of anti-HCV in post-transfusion hepatitis by a thirdgeneration ELISA. Vox Sang 1995;68:15-18 (and personal communication with the authors).

3 Barbara J, Briggs M. Hepatitis of the non-A, non-B type following blood transfusion in the north London region. Med Lab Sci 1981;38:423-6.

4 Ramsay ME, Balogun MA, Collins M, et al. Laboratory surveillance of hepatitis $C$ virus infection in England and Wales: 1992 to 1996. Commun Dis Public Health 1998;1:8994.

5 MacLennan S, Moore MC, Hewitt PE, et al. A study of anti-hepatitis C positive blood donors: the first year of screening. Transfusion Med 1994;4:125-33.

6 Salisbury D, Begg NT, eds. Immunisation against infectious disease. London: HMSO, 1996.

7 Flanagan P, Snape T. Nucleic acid technology (NAT) testing and the transfusion service: a rationale for the implementation of minipool testing. Transfusion Med 1998; 8:9-13.

8 Regan FAM, Barbara JAJ, Bailey $M$, et al. Study to determine the incidence of transfusion transmitted infection in recipients of blood transfusion [abstract]. Transfusion Med 1996;6(suppl 2):5.

9 Hewitt PE, Kendall B, Barbara JAJ. Hepatitis A transmitted by red cell transfusion [abstract]. Transfusion Med 1997; 7 (suppl 1):48.

10 HIV infection transmitted through transfusion. CDR Weekly 1997;7:137.

11 Ricketts MN, Cashman NR, Stratton EE, et al. Is Creutzfeldt-Jacob disease transmitted in blood? Emerg Infect Dis 1997;3:155-63. 